\title{
La lecture des œuvres complètes en contexte scolaire au Québec
}

État des lieux

Reading whole works in Quebecker schools Review

La lectura de obras completas en un contexto escolar en Quebec Estado de la cuestión

Olivier Dezutter, Julie Babin, Marcel Goulet et Lise Maisonneuve

\section{OpenEdition}

\section{Journals}

Édition électronique

URL : https://journals.openedition.org/ries/2690

DOI : 10.4000/ries.2690

ISSN : 2261-4265

Éditeur

France Education international

Édition imprimée

Date de publication : 1 décembre 2012

Pagination : 111-120

ISSN : 1254-4590

Référence électronique

Olivier Dezutter, Julie Babin, Marcel Goulet et Lise Maisonneuve, "La lecture des œuvres complètes en contexte scolaire au Québec », Revue internationale d'éducation de Sèvres [En ligne], 61 | décembre 2012, mis en ligne le 06 février 2015, consulté le 05 juillet 2021. URL : http://journals.openedition.org/ ries/2690 ; DOI : https://doi.org/10.4000/ries.2690 


\section{La lecture des œuvres complètes en contexte scolaire au Québec}

\section{État des lieux}

\section{Olivier Dezutter \\ Julie Babin \\ Marcel Goulet \\ Lise Maisonneuve}

Les occasions de contact avec la littérature à l'école prennent de nos jours des formes multiples: lecture de textes sous diverses modalités, activités de création, activités d'adaptation, rencontres avec des auteurs, etc. De toutes ces formes, c'est la lecture de livres qui retient ici notre attention. À l'échelle de la longue histoire du système scolaire, cette pratique est relativement récente: pendant longtemps, la lecture de la littérature en contexte scolaire était limitée à la lecture d'extraits, rassemblés en anthologies spécialisées ou intégrés aux manuels scolaires. Pour que les livres en tant qu'objets de lecture soient introduits en classe, il a fallu attendre que se développent des éditions à prix modique, comme les éditions de poche au milieu du XXe siècle (Chervel, 2006). Ces formats ont surtout été commercialisés à partir des années cinquante (Legendre, 2010) et ont ainsi permis l'accès à de vastes corpus d'œuvres classiques et contemporaines, dont certaines publiées dans des collections spécifiquement dédiées aux lecteurs d'âge scolaire.

\section{LA PLACE DES GUVRES COMPLÈTES DANS LE CURRICULUM QUÉBÉCOIS}

La masse des œuvres actuellement disponibles oblige les enseignants des différents ordres scolaires à procéder à des opérations de sélection de corpus, guidés par des consignes ministérielles plus ou moins contraignantes selon les pays. Au Québec ${ }^{1}$, les plus récents programmes officiels pour le primaire et le secondaire réaffirment l'importance de la lecture de la littérature, considérée tout

\footnotetext{
1. Nos travaux portent uniquement sur le secteur francophone, qui représente le secteur majoritaire de l'éducation au Québec. La loi 101 votée en 1977 rend obligatoire la scolarisation en français des enfants de parents francophones du Québec ou des immigrants. Seuls les parents de citoyenneté canadienne et dont l'un des deux a vécu la majeure partie de la scolarité en anglais peuvent scolariser leurs enfants dans les commissions scolaires anglophones.
} 
à la fois comme instrument de découverte de la langue, élément de construction identitaire et occasion de découverte de soi et du monde (Gouvernement du Québec, 2001 ; 2004). Dans cette optique, la lecture de livres est considérée comme primordiale, et un nombre minimal d'œuvres à faire lire aux élèves est prescrit : au secondaire, dix œuvres complètes par année ; au collégial ${ }^{2}$, au moins deux œuvres par cours de soixante heures, pour un total de huit œuvres au terme de trois cours obligatoires.

Des balises très larges sont fournies pour la sélection des auteurs et des titres. Au secondaire, les seules contraintes à respecter par les enseignants tiennent au respect d'une certaine diversité : variété d'auteurs et d'univers, équilibre entre les œuvres écrites par des hommes et celles écrites par des femmes, priorité aux œuvres contemporaines avec ouverture sur celles du passé, accent sur les œuvres de la littérature québécoise par rapport à celles de France ou du reste du monde (Gouvernement du Québec, 2004, 2009b). Au niveau collégial, la lecture des œuvres complètes s'inscrit dans le cadre des trois cours de Français, langue et littérature, où les œuvres traduites sont exclues. La sélection s'effectue selon les critères suivants: "Les titres ont marqué l'histoire de la littérature d'expression française ; ils appartiennent à des époques différentes; ils touchent aux quatre principaux genres littéraires (poésie, théâtre, discours narratif, essai) [...] Ces choix assurent une place équilibrée à la littérature québécoise » (Gouvernement du Québec, 2009a, p. 7).

Considérant l'importance accordée à la lecture des œuvres et la grande liberté laissée aux enseignants pour leur sélection, nous avons institué depuis 2004 un programme de recherche visant à dresser un portrait des pratiques des enseignants québécois des trois ordres d'enseignement (primaire, secondaire et collégial) relativement aux choix des œuvres et aux activités menées autour de ces dernières. À la suite de Canvat (1999), nous considérons en effet que les questions relatives à l'accès aux corpus, au choix des textes à faire lire aux élèves, de même que celles portant sur les types d'activités et sur les finalités à poursuivre touchent à des enjeux majeurs d'ordre cognitif, éducatif et sociopolitique.

Notre programme de recherche, à visée descriptive, est fondé sur trois principaux niveaux d'information. Premièrement, des questionnaires ont été remplis par 241 enseignants du dernier cycle du primaire, 392 enseignants du secondaire et 135 enseignants du niveau collégial. Deuxièmement, des entretiens individuels ont été réalisés avec 45 enseignants des niveaux primaire et secondaire et 18 enseignants du collégial. Pour le niveau collégial, nous avons aussi examiné 413 plans de cours fournis à l'automne 2009 par les responsables de département de français ou les directions des études. Même si notre échantillon

2. Propre au système éducatif québécois, le collégial est un niveau de formation pré-universitaire ou technique qui suit le secondaire, après la onzième année de formation obligatoire. Cette formation est assurée dans les collèges d'enseignement général et professionnel (CÉGEP). 
de répondants a été constitué à partir d'une méthode non probabiliste et que nous n'aspirions pas à une représentativité statistique, l'échantillon total de répondants à l'enquête par questionnaire reflète un grand nombre de caractéristiques de la réalité scolaire au Québec, notamment pour la proportion d'écoles publiques et privées, l'équilibre entre les milieux ruraux et urbains, ou l'expérience dans l'exercice de la profession.

En prenant appui sur les informations fournies à travers les différents outils de collecte de données, nous fournissons ici un état des lieux synthétique des pratiques rapportées par les enseignants à propos, d'une part, des représentations qu'ils se font de leur rôle et des finalités associées à la pratique de lecture des œuvres complètes ${ }^{3}$ et, d'autre part, du nombre d'œuvres lues, des critères de sélection ainsi que des corpus retenus.

\section{LES FINALITÉS DE LA LECTURE DES EUVRES COMPLÈTES}

Les enseignants interviewés pour notre étude se sont prononcés sur les raisons - au-delà des obligations curriculaires - de travailler les œuvres complètes en classe. En reprenant la typologie proposée par Richard (2006), quatre finalités résument l'enseignement de la littérature : celles d'ordre cognitivo-langagier, d'ordre psycho-affectif, d'ordre esthético-culturel et d'ordre social et politique. Dans cette perspective, des entretiens réalisés se dégage une préoccupation des enseignants du primaire et du secondaire pour le développement du plaisir de lire (finalité psychoaffective). Et si cette visée est partagée par un tiers des enseignants du collégial rencontrés, ces derniers évoquent en priorité la nécessité de développer une culture littéraire de base (finalité esthético-culturelle) : «La culture, ça sert à [...] avoir des références, à ouvrir l'horizon ». Neuf d'entre eux considèrent d'ailleurs que c'est leur rôle de faire découvrir les œuvres considérées comme majeures aux étudiants. Plusieurs (dix sur dix-huit) parlent également de l'importance d'apprendre aux jeunes à mieux lire, à saisir l'importance du langage (finalité cognitivo-langagière), mais cette finalité n'est pas explicitement liée aux ouvres complètes.

\section{LE NOMBRE D'çUVRES À LIRE}

Quantitativement, les informations recueillies permettent de constater que la lecture de plusieurs œuvres complètes est bien présente dans les classes des trois niveaux scolaires examinés. L'ampleur des ouvres lues ne peut pas, a priori, se comparer d'un niveau à l'autre puisque se trouvent plusieurs albums

3. Dans le cadre de cette étude, la définition retenue d'œuvres complètes est celle proposée par Dezutter et al. (2007), soit les œuvres comme « les romans, les recueils de poésie, de nouvelles, de contes, les textes de pièces de théâtre, les biographies, les essais, les ouvrages documentaires, les bandes dessinées et les albums illustrés dont on n'exploite pas que des extraits » (p. 85). 
parmi les œuvres lues au primaire, mais il n'en reste pas moins que la pratique de lecture qui nous intéresse peut être considérée comme un habitus installé dès le primaire. Au dernier cycle du primaire, un enseignant sur cinq environ (17\%) affiche des exigences très élevées, avec un programme de lecture qui comporte dix œuvres ou plus par année.

Le nombre moyen d'œuvres données à lire par an au secondaire $(4,72)$ est légèrement supérieur au standard de quatre œuvres imposé par les instructions officielles de 1995 (Gouvernement du Québec, 1995), mais rappelons que ce nombre minimal a été augmenté à dix dans le plus récent Programme de formation de l'école québécoise (Gouvernement du Québec, 2004, 2009b). Ainsi, sachant que $63,3 \%$ des enseignants du secondaire interrogés ont des exigences inférieures ou égales au standard officiel de 1995, on peut douter de l'atteinte des objectifs des dernières instructions par tous les enseignants. De surcroît, il apparaît que le nombre moyen d'œuvres données à lire par les enseignants des écoles secondaires privées (5,6 œuvres) - que fréquentent généralement des élèves sélectionnés - dépasse celui des écoles publiques. Les meilleurs élèves ayant l'occasion de découvrir un plus grand nombre d'œuvres, on peut penser que l'image de la lecture en tant qu'objet de distinction intellectuelle reste marquante. Au niveau collégial, enfin, le seuil quantitatif fixé par le ministère - huit œuvres pour l'ensemble des trois cours - semble respecté dans l'ensemble, puisque la moyenne établie pour les professeurs de notre échantillon est autour de trois ouvres par cours.

\section{LECTURES LIBRES OU IMPOSÉES ?}

$\mathrm{Au}$ fil de la scolarité, une différence nette existe en ce qui concerne la liberté de choix laissée ou non aux élèves dans la sélection des œuvres. Alors qu'un enseignant sur deux $(51,9 \%)$ à la fin du primaire laisse une liberté totale de choix ou n'impose que quelques critères généraux de sélection afin, par exemple, que les élèves ne se limitent pas à un seul auteur ou à un seul genre, le pourcentage d'enseignants du secondaire qui agissent de la sorte n'est que de $16 \%$. À ce niveau de la scolarité, plus des trois quarts des enseignants $(83,4 \%)$ imposent la totalité ou la plupart des œuvres. Cette réalité s'explique en partie par le fait que, selon la Loi sur l'instruction publique (Gouvernement du Québec, 2012), il n'est pas possible de demander une contribution aux parents d'élèves du primaire et du secondaire pour l'achat d'ouvrages scolaires, dont les ouvrages littéraires font partie. Le choix des enseignants est donc restreint par les possibilités offertes par la bibliothèque scolaire, souvent constituée en partie de séries d'exemplaires des mêmes ouvrages.

Dans les cours de français au collégial, la sélection des œuvres est faite presqu'exclusivement par les enseignants : 92,9\% de ceux interrogés dans le questionnaire imposent tous les titres. L'enjeu majeur semble donc de contribuer 
à faire connaître "les œuvres et les textes littéraires étudiés [qui] ont marqué l'histoire de la littérature » (Gouvernement du Québec, 2009a, p. 6) plutôt que de laisser le choix aux étudiants de lire n'importe quelle œuvre, comme cela peut se faire dans le secondaire.

\section{LES CRITÈRES DE SÉLECTION DES GUVRES}

Pour connaître ce qui guide généralement les enseignants dans le choix des œuvres à proposer ou à imposer en lecture, nous leur avons soumis une liste fermée de critères à ordonner par ordre d'importance, adaptés selon les spécificités des différents niveaux de scolarité. Pour les enseignants du dernier cycle du primaire et du secondaire interrogés, le critère principal de choix pour $90,3 \%$ des sujets de notre échantillon réside dans le plaisir et l'intérêt qu'ils présupposent que l'œuvre suscitera chez les élèves. Le plaisir et l'intérêt de l'enseignant sont aussi pris en compte, un peu plus au secondaire $(87,6 \%)$ qu'au primaire $(79,9 \%)$; les enseignants semblent donc généralement faire découvrir des œuvres qu'ils apprécient. Pour une bonne majorité d'entre eux - et ici aussi davantage pour les enseignants du secondaire (82,8 \% par rapport à 70,9\% des enseignants du primaire) - les œuvres sont aussi choisies en fonction de leur importance au regard d'une culture littéraire de base. Comme indiqué antérieurement à propos des limitations légales, des contraintes matérielles pèsent également sur les choix, tant au primaire qu'au secondaire : le coût (pour $64,7 \%$ des enseignants) et le nombre suffisant de livres disponibles à la bibliothèque scolaire (pour 60,2 \% des enseignants) influencent la sélection.

Conformément aux orientations des cours de français au collégial et aux critères de sélection balisés par le ministère, ce sont les dimensions littéraires et patrimoniales qui constituent les critères les plus souvent utilisés par les enseignants de ce niveau : la valeur de l'œuvre reconnue par l'institution littéraire (95,9\% des enseignants) et son appartenance - du point de vue de l'enseignant - à une culture littéraire de base (92,9\%). À ce propos, il faut savoir que les enseignants du collégial, formés essentiellement en littérature et souvent diplômés au niveau de la maîtrise dans cette discipline, ne présentent pas le même profil de formation initiale que les enseignants du secondaire, formés surtout sur les plans didactique et pédagogique.

Un autre critère de sélection peut s'expliquer par la présence d'une épreuve ministérielle obligatoire sanctionnant les études collégiales et prenant la forme d'une dissertation littéraire ${ }^{4}$ qui permet à l'élève de démontrer « des

4. L'existence de cette épreuve pèse lourd sur les pratiques des enseignants et limite fortement les occasions d'engager les étudiants dans d'autres formes d'écrits sur la littérature, dont les écrits de création. Des activités de création littéraire n'ont été repérées que dans moins de la moitié (44\%) des plans de cours examinés. 
compétences suffisantes en lecture [...] pour comprendre des textes littéraires » (Gouvernement du Québec, 2010). Dans ce contexte, le repérage de procédés stylistiques est inscrit au programme. Le fait qu'une œuvre permette d'illustrer un procédé particulier d'écriture constitue dès lors sans surprise, pour 84,7 \% des répondants à notre questionnaire, un critère " souvent » ou « toujours » pris en compte.

\section{LE CORPUS}

\section{Les genres}

$\mathrm{Au}$ primaire comme au secondaire, le roman domine largement. En continuité avec la finalité psycho-affective évoquée précédemment, les enseignants optent pour des romans censés rejoindre les goûts des jeunes lecteurs et axés sur le suspense (roman fantastique, policier ou d'aventures). Le théâtre en tant qu'œuvre complète n'occupe qu'une maigre place $(5,4 \%$ des titres cités dans les questionnaires), et la bande dessinée est pratiquement absente (1,2\%). La diversité générique est plus grande au niveau collégial, conformément aux instructions officielles qui demandent d'aborder les quatre genres principaux. Si le genre narratif domine (91,9\% des plans de cours), deux étudiants du collégial sur trois ont à lire une pièce de théâtre en intégralité $(68,7 \%$ des plans de cours) et un étudiant sur cinq un recueil de poésie $(19,5 \%)$. La lecture de l'essai reste marginale $(5,9 \%)$.

\section{La place de la littérature québécoise}

Grâce à l'important soutien des instances gouvernementales provinciales, soucieuses de développer l'édition en français dans le contexte linguistique propre au Québec, le marché de la littérature québécoise est extrêmement riche et diversifié et le secteur de la littérature jeunesse, particulièrement foisonnant. Les livres édités au Québec coûtent aussi moins cher que ceux édités ailleurs. Ajoutés aux orientations ministérielles qui exigent d'accorder en classe une place appréciable à la littérature québécoise, ces éléments de contexte expliquent en grande partie la présence quantitativement importante des auteurs québécois au sein des corpus imposés : au primaire, la littérature québécoise représente $70 \%$ des titres cités par les enseignants de notre étude.

Le palmarès des auteurs mis au programme par les enseignants interrogés (tableau 1 ci-contre) met en lumière, au secondaire, six auteurs québécois sur les huit les plus lus. Marineau et Demers publiant à la fois dans les collections pour la jeunesse et dans les collections grand public, cela explique en grande partie leur présence en tête du palmarès établi pour l'ensemble du secondaire. 
Tableau 1

Palmarès des auteurs lus au secondaire et au collégial

\begin{tabular}{|c|c|c|c|}
\hline \multicolumn{2}{|c|}{$\begin{array}{c}\text { Secondaire } \\
\text { (sur un total de } 458 \text { auteurs) }\end{array}$} & \multicolumn{2}{|c|}{$\begin{array}{c}\text { Collégial } \\
\text { (sur un total de } 243 \text { auteurs cités } \\
\text { dans les plans de cours) }\end{array}$} \\
\hline Auteurs & $\mathrm{Nb}$ & Auteurs & $\mathrm{Nb}$ \\
\hline Michèle Marineau* & 80 & Molière & 114 \\
\hline Dominique Demers* & 72 & Voltaire & 60 \\
\hline Agatha Christie & 51 & Michel Tremblay* & 54 \\
\hline Chrystine Brouillet* & 44 & Guy de Maupassant & 50 \\
\hline Robert Soulières* & 42 & Victor Hugo & 45 \\
\hline Michel Tremblay* & 40 & & \\
\hline Denis Côté ${ }^{*}$ & 38 & & \\
\hline Alexandre Jardin & 34 & & \\
\hline
\end{tabular}

*Auteur(e) québécois(e).

$\mathrm{Au}$ second cycle du secondaire, la proportion d'auteurs québécois diminue (de 67,4\% au premier cycle à 43,8 \%), laissant plus de place à la littérature francophone hors Québec et aux œuvres traduites. Au collégial, il faut noter que l'un des trois cours obligatoires est consacré entièrement à la littérature québécoise (même si des auteurs québécois peuvent aussi être inscrits au programme des deux autres cours, centrés sur la littérature française). Cela dit, tous cours confondus, les données recueillies dans les plans de cours montrent que 42,4 \% des enseignants inscrivent au moins une œuvre de littérature québécoise au programme. Compte tenu de l'émergence relativement récente de cette littérature, les œuvres sont largement issues de la seconde moitié du vingtième siècle $(86,5 \%$ des titres cités).

\section{Les auteurs et les titres}

Il faut noter que la dispersion des auteurs comme des titres est beaucoup plus grande au secondaire qu'au collégial. Pour le secondaire, les 392 enseignants participants ont déclaré, dans les œuvres imposées, 779 titres et 458 auteurs différents. On est donc très loin d'un consensus autour de quelques " incontournables ». Les deux titres recueillant le plus de mentions - La route de Chlifa et Le Petit Prince - ne sont imposés chacun que par 1,1\% des enseignants et sont lus plus souvent au premier cycle. Les enseignants du secondaire semblent donc profiter pleinement de la liberté laissée par le ministère en matière de corpus à faire découvrir aux élèves. 
Tableau 2

Palmarès des titres lus au deuxième cycle du secondaire et au collégial

\begin{tabular}{|c|c|c|c|}
\hline \multicolumn{2}{|c|}{$\begin{array}{c}2^{\mathrm{e}} \text { cycle du secondaire } \\
\text { (sur } 1353 \text { œuvres imposées) }\end{array}$} & \multicolumn{2}{|c|}{$\begin{array}{c}\text { Collégial } \\
\text { (sur } 1225 \text { œuvres imposées) }\end{array}$} \\
\hline Titres & $\mathrm{Nb}$ & Titres & $\mathrm{Nb}$ \\
\hline Des souris et des hommes & 25 & Candide & 41 \\
\hline Le parfum & 20 & Don Juan & 34 \\
\hline$C^{\prime}$ est pas moi, je le jure !* & 20 & Tristan et Iseult & 32 \\
\hline Dix petits nègres & 19 & Le Dernier jour d'un condamné & 31 \\
\hline L'alchimiste & 18 & Les Fleurs du Mal & 20 \\
\hline \multirow[t]{2}{*}{ La vie devant soi } & 17 & Le Bourgeois gentilhomme & 19 \\
\hline & & À toi, pour toujours, ta Marie-Lou ${ }^{*}$ & 17 \\
\hline
\end{tabular}

* Fuvre québécoise.

Nous avons déjà indiqué que la dimension patrimoniale est clairement affichée dans les orientations des cours de Français, langue et littérature au collégial, ce qui peut expliquer un corpus beaucoup plus resserré à ce niveau : 398 titres ont été évoqués dans les 413 plans de cours examinés, pour un total de 243 auteurs différents. Il faut ajouter que, lorsqu'il s'agit de faire lire des ouvres du passé, le consensus se fait plus facilement autour d'un noyau d'auteurs et de titres - sans doute sous l'influence de la tradition scolaire et des instances de légitimation.

À titre comparatif, le tableau 2 indique les titres d'œuvres les plus souvent sélectionnés par les enseignants du dernier cycle du secondaire et par ceux du collégial.

Comme on l'a indiqué antérieurement, les œuvres québécoises ne sont plus majoritaires à la fin du secondaire, et une place est faite à des œuvres marquantes de la littérature française ou traduite. Quant aux œuvres françaises inscrites au palmarès du collégial, leur présence importante s'explique par les exigences des deux premiers cours de français, qui couvrent la littérature française du Moyen Âge au XX ${ }^{\mathrm{e}}$ siècle.

Notre recherche, effectuée auprès de centaines d'enseignants québécois, montre des pratiques distinctes autour de l'œuvre complète selon le niveau scolaire. Au primaire et au secondaire, les enseignants se préoccupent d'abord de la finalité psychoaffective de la littérature : ils priorisent l'intérêt des élèves en leur donnant souvent des choix et en proposant des titres variés, surtout des romans québécois. Au collégial, probablement influencés par le programme d'étude, les enseignants visent surtout la finalité esthético-culturelle en imposant 
en majorité des œuvres considérées comme majeures d'auteurs français et québécois et de genres divers, qui permettraient de développer chez les étudiants une culture littéraire et d'étudier un certain nombre de procédés stylistiques.

\section{BibliographiE}

CANVAT K. (1999) : "A propos du corpus littéraire : questions et propositions ». Français 2000, 163/164, 30-40.

CHERVEL A. (2006) : Histoire de l'enseignement du français du XVII au XXe siècle. Paris : Éditions Retz.

DEZUTTER O., LARIVIÈRE I., BERGERON M.-D. et MORISSETTE C. (2007). Les pratiques déclarées des enseignants québécois dans la sélection et l'exploitation des oeuvres complètes inscrites au programme de lecture des élèves. In E. Falardeau, C. Fisher, C. Simard et N. Sorin (dir.), La didactique du français : les voies actuelles de la recherche (p. 83-100). Lévis, QC : Les Presses de l'Université Laval.

GOUVERNEMENT DU QUÉBEC (1995) : Programme de français, langue d'enseignement. Québec : Ministère de l`Éducation du Québec. http://www.mels.gouv.qc.ca/DGFJ/dp/ programmes_etudes/secondaire/pdf/prfrans.pdf.

- (2001) : Programme de formation de l'école québécoise (éducation préscolaire et enseignement primaire). Québec: Ministère de l'Éducation, du Loisir et du Sport. http://www.mels.gouv.qc.ca/dgfj/dp/programme_de_formation/primaire/pdf/ prform2001/prform2001.pdf [consulté le 21 mars 2011].

- (2004) : Programme de formation de l'école québécoise ( $1^{\text {er }}$ cycle du secondaire). Québec : Ministère de l'Éducation, du Loisir et du Sport. http://www.mels.gouv.qc.ca/ sections/programmeFormation/secondaire1/ [consulté le 10 août 2012].

- (2009a) : Formation générale commune, propre et complémentaire aux programmes d'études conduisant au diplôme d'études collégiales. Québec, QC: Ministère de l'Éducation, du Loisir et du Sport. http ://www.mels.gouv.qc.ca/sections/publications/ index.asp ?page=fiche\&id=1134 [consulté le $1^{\text {er }}$ août 2011].

- (2009b) : Programme de formation de l'école québécoise ( $2^{e}$ cycle du secondaire). Québec : Ministère de l'Éducation, du Loisir et du Sport. http://www.mels.gouv.qc.ca/ sections/programmeFormation/secondaire2/medias/PFEQ_FrancaisLangue Enseignement.pdf [consulté le 2 août 2011].

- (2010) : Épreuve uniforme de français du collégial : Langue d'enseignement et littérature. http://www.mels.gouv.qc.ca/ens-sup/ens-coll/Eprv_uniforme/Mfrancais.asp [consulté le 21 mars 2011].

- (2012) : Loi sur l'instruction publique. Québec : Éditeur officiel du Québec. http:// www2.publicationsduquebec.gouv.qc.ca/dynamicSearch/telecharge. php?type=2\&file=/I_13_3/I13_3.html [consulté le 07 août 2012].

LEGENDRE B. (2010): Les débuts de l'édition de poche en France : entre l'industrie et le social (1953-1970). Mémoires du livre, 2(1).

RICHARD S. (2006) : Analyse des discours didactiques concernant les finalités de l'enseignement de la littérature au secondaire de 1970 à 2004. In E. Falardeau, C. Fisher et N. Sorin (dir.), Le français, discipline singulière, plurielle ou transversale (p.). Actes du $9^{\text {e }}$ colloque de l'Association internationale pour le développement de la recherche en didactique du français. http://www.colloqueairdf.fse.ulaval.ca/fichier/ Communications/Suzanne-Richard.pdf [consulté le 23 mars 2011]. 
